\title{
The Research of Magneto-Rheological Fluid Yield Stress Model
}

\author{
Meng Ji, Yiping Luo* \\ Shanghai University of Engineering Science, Shanghai, China \\ Email:*949299114@qq.com
}

How to cite this paper: Ji, M. and Luo, Y.P. (2018) The Research of Magneto-Rheological Fluid Yield Stress Model. Open Access Library Journal, 5: e4643.

https://doi.org/10.4236/oalib.1104643

Received: May 7, 2018

Accepted: June 5, 2018

Published: June 8, 2018

Copyright $\odot 2018$ by authors and Open Access Library Inc.

This work is licensed under the Creative Commons Attribution International License (CC BY 4.0).

http://creativecommons.org/licenses/by/4.0/

\begin{abstract}
Magnetorheological Fluid (MRF), as an advanced and smart material which was controlled by magnetic field, was a kind of stable suspension in which magnetic particle dissolved in base fluid. The yield stress, one of main performance parameters of MRF, was the demarcation point between liquid and solid. At present, the yield stress calculation model did not have a uniform standard. The research on yield stress model was significant to the research on MRF. First, the research was based on the MRF characteristic and the research status of MRF sheer yield stress; second the classic dipole model, local field dipole model, polarized pellet model, continuous models on the average had been calculated and compared. The classic dipole model and local field dipole model had a well ability to describe the yield stress of MRF.
\end{abstract}

\section{Subject Areas \\ Functional Materials}

\section{Keywords}

MRF, Classic Dipole Model, Local Field Dipole Model, Polarized Pellet Model, Continuous Models

\section{Introduction}

Magnetorheological fluid (MRF), as a new advanced material, was a type of stable suspension liquid which was composed of magnetizable particle and base solution. The MRF had a reversible ability that it could converse fluid into semisolid in millisecond in a changeable and stable magnetic field. The apparent viscosity increased $105 \sim 106$ times and the maximum yield stress increased to $50 \sim 100 \mathrm{kPa}$. Using MRF could solve the problem that traditional machine cannot solve. This excellent ability is given a vast prospect of MRF application. 
In automobile, mechanical precision polishing and other aspects had a large market [1] [2].

Magnetorheological fluid consists of magnetic particles, the base fluid and additives respectively. Magnetic particles were the main part of the performance, the base fluid was as carrier to carry magnetic particles and additive is to improve performance [3] [4].

1) Magnetic particles

There exists a main tendency that the magnetic particles were hydroxyl iron powder, iron-cobalt alloy and iron-nickel alloy in nowadays research. Iron-cobalt alloy and iron-nickel alloy had a batter performance and were used in research application, but the high price was not ignored. The cost of hydroxyl iron powder was much low and it had an easier preparation technology. So hydroxyl iron powder was the best choice in MRF preparation technology and the performance is satisfied in the meantime. In order to guarantee MRF having a high yield stress, the magnetic particle had following characters:

(1) High permeability. The ability of magnetic energy level was depended on the permeability of magnetic-particle; a high permeability is a guarantee of yield stress.

(2) Low coercivity. Low coercive force for MRF can improve demagnetization and transfer, and transfer the reversible process.

(3) Magnetic particle size. Results indicate that: if magnetic particles diameter in a suitable range increased, the yield stress will increase. But beyond the suitable scope, relationship could no longer exist. So it was also important to select a suitable diameter size, usually 1 - 10 microns.

2) Base fluid

Base fluid, as a carrier, had two kinds of magnetic carrier fluid: the nonmagnetic carrier fluid and magnetic carrier fluid which had been widely applied. The nonmagnetic carrier fluid was embodied in Bingham plastic fluids and Newtonian fluid respectively in magnetic field or without magnetic field. At present the common base products included silicone oil, water, and synthetic oil. Silicone oil was the most widely used. It was colorless, tasteless, high stability and low price. Base fluid usually had the following features:

(1) Low viscosity. Fluid of low viscosity is the important guarantee of MRF viscosity low zero field.

(2) Good stability, corrosion resistance. The stability of the base fluid directly affected the stability of magnetorheological fluid. Corrosion resistance was also in the bad environment to ensure safety work.

(3) High density. Base fluid density is high, which can effectively prevent subsidence problems and improve the comprehensive performance.

3) Additive

Additive can improve the performance of MRF, such as:

(1) Reduce the settle-ability.

(2) The lubrication effect. Additive can prevent the sticky between solid particle and maintain the liquid homogeneity. 
(3) Improve magnetic susceptibility. Improve rheological property and enhance the polarization performance of magnetic particle.

The research status of MRF yield stress testing device

Ginder [5] research result based on utilizing finite element method indicated that maximum yield stress is proportional to the magnetic saturation magnetization intensity of particle. Weiss [6] indicated that yield stress decrease with temperature increase.

Felt [7] finished the research about the affect of volume fraction and particle size to MRF yield stress and indicated that volume fraction and particle size is proportional to MRF yield stress. Jiang [8] indicated that static yield stress is proportional to particle size.

\section{MRF Yield Stress Calculation Model}

\subsection{The Classic Dipole Model}

In Figure $1(\mathrm{a}), R$ and $\mu_{1}$ was particle radius and permeability, $\mu_{2}$ was permeability of basic liquid, $r$ was straight-line distance, $\varphi$ was magnetic potential. When the magnetic field was $H_{0}$, particle inner and outer magnetic potential had a following relation:

$$
\begin{aligned}
& \nabla^{2} \varphi_{1}=0 \quad(r<R) \\
& \nabla^{2} \varphi_{2}=0 \quad(r>R)
\end{aligned}
$$

Function (2-1) formed Laplace's equations. boundary conditions were:

$$
\begin{gathered}
r=0, \quad \varphi_{1}=0 \\
r=\infty, \varphi_{2}=-H_{0} r \cos \theta \\
r=R, \varphi_{1}=\varphi_{2}, \mu_{1} \frac{\partial \varphi_{1}}{\partial r}=\mu_{2} \frac{\partial \varphi_{2}}{\partial r}
\end{gathered}
$$

Solution of equations:

$$
\begin{gathered}
\varphi_{1}=-\frac{3 \mu_{2}}{\mu_{1}+2 \mu_{2}} H_{0} r \cos \theta \\
\varphi_{2}=\left(-r+\frac{\mu_{2}-\mu_{1}}{\mu_{1}+2 \mu_{2}} \frac{R^{3}}{r^{2}}\right) H_{0} \cos \theta
\end{gathered}
$$

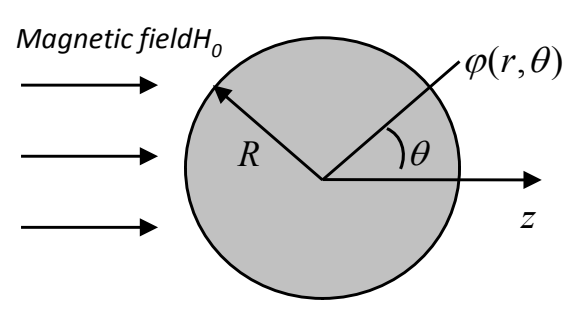

(a)

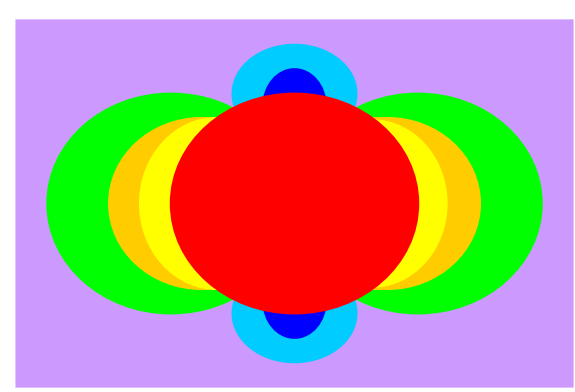

(b)

Figure 1. Magnetic field distribution of classic dipole model. (a) Single particle model diagram under magnetic field; (b) Magnetic field map of the dipole model. 
The relation between magnetic field and magnetic potential was $H=-\nabla \varphi$, the expression of particle inner and outer magnetic field:

$$
\begin{gathered}
H_{1}=\frac{3 \mu_{2}}{\mu_{1}+2 \mu_{2}} H_{0}\left(\cos \theta e_{r}-\sin \theta e_{\theta}\right) \\
H_{2}=\left(1-2 \frac{\mu_{2}-\mu_{1}}{\mu_{1}+2 \mu_{2}} \frac{R^{3}}{r^{3}}\right) H_{0} \cos \theta e_{r}-\left(1+2 \frac{\mu_{2}-\mu_{1}}{\mu_{1}+2 \mu_{2}} \frac{R^{3}}{r^{3}}\right) H_{0} \sin \theta e_{\theta}
\end{gathered}
$$

Due to $e_{z}=e_{r} \cos \theta-e_{\theta} \sin \theta$, we assumed $k_{1}=\frac{3 \mu_{2}}{\mu_{1}+2 \mu_{2}}, \quad k_{2}=\frac{\mu_{2}-\mu_{1}}{\mu_{1}+2 \mu_{2}}$, the Equation (2-4) simplify:

$$
\begin{gathered}
H_{1}=k_{1} H_{0} e_{z} \\
H_{2}=H_{0} e_{z}-\left(2 \cos \theta e_{r}+\sin \theta e_{\theta}\right) k_{2} H_{0} \frac{R^{3}}{r^{3}}
\end{gathered}
$$

According to Equation (2-5), the inner magnetic field of particle is uniform magnetic field, particle outer magnetic field $H_{2}$ is the first part, $H_{0} e_{z}$ is a outer uniform magnetic field, the second part is addition magnetic field of MRF magnetization, The classic dipole model inside and outside the magnetic field distribution was shown in Figure 1(b).

$$
\begin{aligned}
m & =\frac{4 \pi \mu_{2}\left(\mu_{1}-\mu_{2}\right) R^{3}}{\mu_{1}+2 \mu_{2}} H_{0}=-4 \pi \mu_{2} k_{2} R^{3} H_{0} \\
H_{2} & =H_{0} e_{z}+\frac{1}{4 \pi \mu_{2} r^{3}}\left(2 m \cos \theta e_{r}+m \sin \theta e_{\theta}\right) \\
& =H_{0}+\frac{1}{4 \pi \mu_{2}}\left(\frac{3(m \cdot r) r}{r^{5}}-\frac{m}{r^{3}}\right)
\end{aligned}
$$

The Equation (2-6) indicated that single particle crease a magnetic field in uniform magnetic field was equal to a magnetic dipole which dipole moment was $m$ crease a magnetic field.

\subsection{Local Dipole Model}

Figure 2 was shown about local dipole model. $R$ is particle radius, the magnetic dipole moment $m_{i}$ was in centre.

There exists a chaining particle $i$ in uniform magnetic field and its magnetic dipole moment was that:

$$
m_{i}^{\prime}=-3 \mu_{2} k_{2} V H_{\text {loc }}
$$

where, $V$ was particle volume; $H_{l o c}$ was local field of particle $i$; $H$ was the magnetic field creased by other particles in chain.

The magnetic field creased by $n$ magnetized particles was that:

$$
\begin{aligned}
H_{p} & =H_{1}+H_{2}+\cdots+H_{t-1}+H_{t}+\cdots+H_{n} \\
& =\sum_{q=1}^{n-1} \frac{1}{4 \pi \mu_{2}}\left(\frac{3\left(m_{1}^{\prime} \cdot r_{q}\right) r_{q}}{r_{q}^{5}}-\frac{m_{1}^{\prime}}{r_{q}^{3}}\right)
\end{aligned}
$$




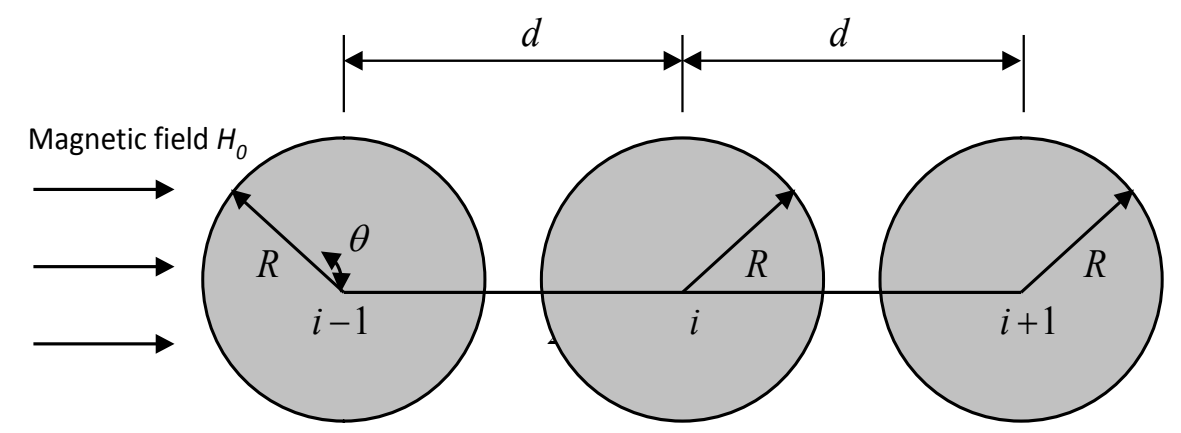

Figure 2. Local field dipole model.

If $n$ showed odd number, the magnetic field creased by centre particle $i$ and alone $H_{0}$ direction was shown that:

$$
H_{p 0}=\frac{4 m_{1}^{\prime}}{4 \pi \mu_{2}} \sum_{q=1}^{(n-1) / 2} \frac{1}{r_{q}^{3}}=\frac{m_{1}^{\prime}}{\pi \mu_{2} d^{3}} \sum_{q=1}^{(n-1) / 2} \frac{1}{q^{3}}
$$

and:

$$
\begin{aligned}
m_{1}^{\prime} & =-3 \mu_{2} k_{2} V\left(H_{0}+H_{p 0}\right) \\
& =-3 \mu_{2} k_{2} V\left(H_{0}+\frac{m_{1}^{\prime}}{\pi \mu_{2} d^{3}} \sum_{q=1}^{(n-1) / 2} \frac{1}{q^{3}}\right)
\end{aligned}
$$

The expression about magnetic dipole moment $m_{1}^{\prime}$ was that:

$$
m_{1}^{\prime}=\frac{-4 \pi \mu_{2} R^{3} k_{2} H_{0}}{1+4 k_{2}\left(\frac{R}{r}\right)^{3} \sum_{q=1}^{(n-1) / 2} \frac{1}{q^{3}}}
$$

Based on the analysis to (2-11), the value of $R$ had a stable value, the value of $(R / d)^{3}$ depend on $d$, when the particle space $\mathrm{d}$ was large, the value of $(R / d)^{3}$ was small, compared to classic dipole model, the moment of dipole $m_{1}^{\prime}$ of local dipole model was very similar with the moment of dipole $m=-4 \pi \mu_{2} k_{2} R^{3} H_{0}$ of local dipole model.

\subsection{Polarized Pellet Model}

As regards polarized pellet model, there exist an assumption that magnetic particle only form isolated chain. The force between particles was utilized magnetic charge to calculate. Lemaire [9] calculated the function based on electric polarization ball:

$$
\tau_{s}=\frac{2 \phi}{3 \pi a^{2}}\left\{3 \mu a^{2} H^{2} f\left(\frac{\mu_{i}-\mu}{\mu_{i}+2 \mu}\right)^{2}\right\}^{m}
$$

where, $\mu, \mu_{i}$ was magnetic particle and MRF tape elongation respectively; $a$ was radius of polarization sphere; $\phi$ was magnetic particle and MRF volume fraction ratio respectively; $H$ was applied uniform magnetic field; $f$ was polarzation coefficient (Figure 3). 


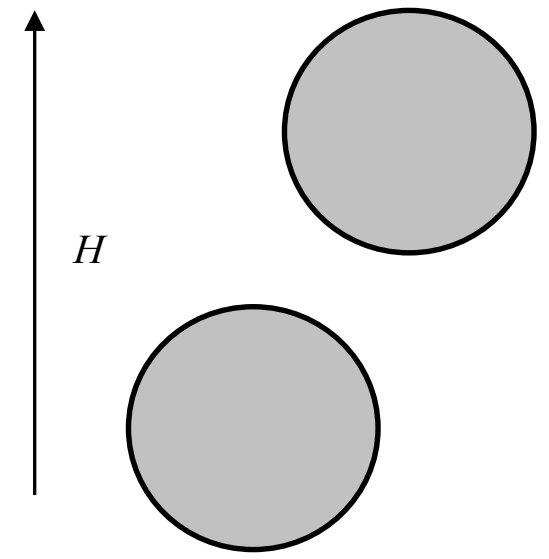

Figure 3. Polarized pellet model.

Lemaire [9] calculated the MRF yield stress based on Equation (2-12), theoretical value tower above experimental value $60 \%$. So, polarized pellet model had an obvious error.

\subsection{Mean Continuous Field Model}

Rosenweig [10] provided a mean continuous field model. He assumed that MRF was a kind of solid with isotropous magnetic susceptibility and yield stress (Figure 4).

Based on Maxwell tensor of stress and asymmetric stress effect, the function stress and strain was that:

$$
\tau=\frac{1}{2} \mu_{0} M_{x} H_{0}=\frac{\mu_{0} H_{0}^{2}}{2}\left(\chi_{\|}-\chi_{\perp}\right) \sin \alpha \cos \alpha
$$

where, $\chi_{\|}, \chi_{\perp}$ was parallel, vertical cylinder structure magnetic susceptibility; $\alpha$ was the angle cylinder axis and magnetic field after yield.

Lemaire [11] calculated based on virtual work principle and mean field model. the expression was shown as following:

$$
\tau=\frac{\partial W(r)}{\partial r}=-\frac{\mu_{0} H_{0}^{2}}{2} \frac{\partial \chi_{y y}}{\partial r}=\frac{\mu_{0} H_{0}^{2}}{2}\left(\chi_{\|}-\chi_{\perp}\right) 2 \sin \alpha \cos ^{3} \alpha
$$

The function (2-14) had a redundant $2 \cos ^{2} \alpha$ compared with function (2-13) because the calculation method may not be suitable for anisotropic material. The model ignored the magnetic concentrate effect between particles, so theoretical value will be lower than the test value.

\section{Conclusions}

According to the 4 models analyzed above, we can make a conclusion that: (1) The classic dipole model had a concise and convenient expression, but it was only used when particle space was far enough. If particle space was near, the error could be out of control. (2) Compared to the classic dipole model, local dipole model had a better accuracy in computation because of effect of other magnetic particle. It had a defeat that when the particle space was very near, the 


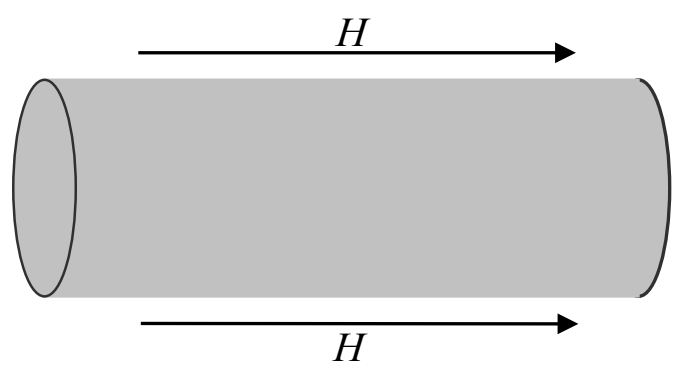

Figure 4. Mean continuous field model.

accuracy decreased because the moment of dipole had an influence by other particle and decentralization effect. (3) Polarized pellet model could not consider the character of magnetic particle non-linearity and the error is obvious. (4) Mean continuous field model ignored the magnetic concentrate effect and the calculated value is lower than test value. Based on the four yield stress calculation model, we could make a reasonable hypothesis to analyze the major influence factor. A suitable assumption was a key to calculate the MRF yield stress.

\section{References}

[1] Bossis, G. and Volkova, O. (2003) Magneto-Rheology: Fluids, Structures and Rheology. Lecture Notes in Physics, 594, 202-230. https://doi.org/10.1007/3-540-45646-5_11

[2] Richter, L., Zipser, L. and Lange, U. (2001) Properties of Magneto-Rheological Fluids. Sensors and Materials, 13, 385-397.

[3] Carlson, J.D. (2002) What Makes a Good MR Fluid. Journal of Intelligent Material Systems and Structure, 13, 431-435. https://doi.org/10.1106/104538902028221

[4] Zhu, X.C., Jing, X.J. and Li, C. (2012) Magnetorheological Fluid Dampers: A Review on Structure Design and Analysis. Journal of Intelligent Material Systems and Structures, 23, 839-873. https://doi.org/10.1177/1045389X12436735

[5] Ginder, J.M. and Davis, L.C. (1994) Shear Stresses in Magnetorheological Fluids: Role of Magnetic Saturation. Applied Physics Letters, 65, 3410-3412. https://doi.org/10.1063/1.112408

[6] Weiss, K.D. and Duclos, T.G. (1994) Controllable Fluids: The Temperature Dependence of Post-Yield Properties. World Scientific, Feldkirch, 20-23.

[7] Felt, D.W., Hagenbuchle, M. and Liu, J. (1996) Rheology of a Magnetorheological Fluid. World Scientific, Singapore, 738-746. https://doi.org/10.1177/1045389X9600700522

[8] Jiang, F.Q., Wang, Z.W. and Wu, J.Y. (1998) Magnetorheological Materials and Their Application in Shock Absorbers. World Scientific, Yonezawa, 494-500.

[9] Lemire, E. and Bossis, G. (1991) Yield Stress and Wall Effects in Magnetic Colloidal Suspensions. Journal of Physics D: Applied Physics, 24, 1473-1477. https://doi.org/10.1088/0022-3727/24/8/037

[10] Rosensweig, R.E. (1995) On Magnetorheology and Electrorheology as Stasus of Unsymmetric Stress. Rheologica Acta, 39, 179-192.

[11] Lemiare, E. and Bossis, G. (1996) Deformation and Rupture Mechanisms of ER and MR Fluids. World Scientific, 11, 143-148. https://doi.org/10.1142/S0217979296001616 